\title{
Postmortem quantitative 1.5-T MRI for the differentiation and characterization of serous fluids, blood, CSF, and putrefied CSF
}

\author{
Wolf-Dieter Zech ${ }^{1} \cdot$ Nicole Schwendener ${ }^{1}$ - Anders Persson ${ }^{2} \cdot$ Marcel J. Warntjes ${ }^{2}$. \\ Fabiano Riva $^{1}$ - Frederick Schuster ${ }^{1,3}$. Christian Jackowski ${ }^{1}$
}

Received: 20 October 2014 / Accepted: 22 June 2015 / Published online: 11 July 2015

(C) Springer-Verlag Berlin Heidelberg 2015

\begin{abstract}
The purpose of the present study was to investigate whether serous fluids, blood, cerebrospinal fluid (CSF), and putrefied CSF can be characterized and differentiated in synthetically calculated magnetic resonance (MR) images based on their quantitative $T_{1}, T_{2}$, and proton density (PD) values. Images from 55 postmortem short axis cardiac and 31 axial brain 1.5-T MR examinations were quantified using a quantification sequence. Serous fluids, fluid blood, sedimented blood, blood clots, CSF, and putrefied CSF were analyzed for their mean $T_{1}, T_{2}$, and PD values. Body core temperature was measured during the MRI scans. The fluid-specific quantitative values were related to the body core temperature. Equations to correct for temperature differences were generated. In a 3D plot as well as in statistical analysis, the quantitative $T_{1}, T_{2}$ and PD values of serous fluids, fluid blood, sedimented blood, blood clots, CSF, and putrefied CSF could be well differentiated from each other. The quantitative $T_{1}$ and $T_{2}$ values were temperature-dependent. Correction of quantitative values to a temperature of $37^{\circ} \mathrm{C}$ resulted in significantly better discrimination between all investigated fluid mediums. We conclude that postmortem 1.5-T MR quantification is feasible to discriminate between blood, serous fluids, CSF, and
\end{abstract}

Wolf-Dieter Zech

wolf-dieter.zech@irm.unibe.ch

1 Institute of Forensic Medicine, University of Bern, Bühlstrasse 20, 3012 Bern, Switzerland

2 Center for Medical Image Science and Visualization (CMIV), Linköping University, Linköping, Sweden

3 Department of Diagnostic, Interventional and Pediatric Radiology, Hospital and University of Bern Inselspital, Freiburgstrasse 10, 3010 Bern, Switzerland putrefied CSF. This finding provides a basis for the computeraided diagnosis and detection of fluids and hemorrhages.

Keywords Postmortem quantitativeMRI · Forensic imaging · Serous fluids $\cdot$ Blood $\cdot$ Putrefaction

$\begin{array}{ll}\text { Abbreviations } \\ \text { AUC } & \text { Area under curve } \\ \text { CSF } & \text { Cerebrospinal fluid } \\ \text { MRI } & \text { Magnetic resonance imaging } \\ \text { PMMRI } & \text { Postmortem magnetic resonance imaging } \\ \text { PMI } & \text { Postmortem interval } \\ \text { RAI } & \text { Radiological alteration index } \\ \text { ROC } & \text { Receiver operating characteristic } \\ \text { ROI } & \text { Region of interest } \\ \text { T } & \text { Tesla } \\ T_{2} \mathrm{~W} & T_{2} \text {-weighted } \\ T_{1} \mathrm{~W} & T_{1} \text {-weighted } \\ \text { PD } & \text { Proton density } \\ \text { TI } & \text { Inversion time } \\ \text { TR } & \text { Repetition time } \\ \text { TE } & \text { Echo time }\end{array}$

\section{Introduction}

Postmortem magnetic resonance imaging (PMMRI) has become a useful adjunct tool to forensic autopsy [1-5]. In addition to its recognized role in the depiction of soft tissue and internal organs, MRI is effective in detecting fluid mediums when the appropriate MRI sequences are used [6-8]. The identification of the nature of the detected fluid medium is highly relevant because it can be an indication of trauma or pathologic processes [9-15]. In conventional MR images, fluid mediums are 
recognized by their signal behavior in different MRI sequences. For instance, fluid mediums appear hypointense in $T_{1}$-weighted $\left(T_{1} \mathrm{w}\right)$ sequences and hyperintense in $T_{2}$-weighted $\left(T_{2} \mathrm{w}\right)$ sequences [16]. Sedimented blood, which can be found in hematomas or hemorrhage effusions in body cavities in the living and deceased, can be recognized easily by its typical twolayered morphological appearance [17]. If blood is not sedimented in the deceased, which occurs in the minority of cases, it is harder to discriminate it from other fluids such as serous fluids or bile in conventional MR images $[17,18]$. The image reader may still be able to discern different fluid types, such as proteinaceous fluids and hemorrhages by different signal intensities in conventional images $[19,20]$. This approach, however, is not always reliable. One approach to discern fluid types and blood reliably may be the use of a recently implemented quantitative MRI sequence. This particular sequence simultaneously quantifies the $T_{1}$ and $T_{2}$ relaxation times and proton density of the imaged tissue, using a multi-slice saturation recovery turbo spin echo sequence with dual-echo acquisition. Based on the measured $T_{1}, T_{2}$, and proton density (PD) values, conventional $T_{1}$-weighted, $T_{2}$-weighted, and PD images can be synthesized with a free choice of echo time (TE), repetition time (TR), and inversion time (TI). Synthetic MRI images are similar to conventional MR images, hence allowing the user to work with MRI images normally. The advantage of this technique, however, is that quantitative measurements can be obtained from the same dataset, where all images are intrinsically co-registrated. The quantified parameters $T_{1}$ (in $\mathrm{ms}$ ), $T_{2}$ (in $\mathrm{ms}$ ), and PD (in $\%$, where $100 \%$ is equivalent to pure water) can be used to characterize fluids in an objective manner by measuring and comparing the three different parameters [21-24]. The purpose of the present study was to investigate whether serous fluids, blood, cerebrospinal fluid (CSF), and putrefied CSF can be differentiated and characterized based on their quantitative $T_{1}, T_{2}$, and PD values.

\section{Materials and methods}

\section{Study subjects}

Blood, serous fluids, CSF, and putrefied CSF from 86 forensic corpses (mean age $51 \pm 17.7$ years, 44 males, 42 females) were analyzed in a prospective study. The inclusion criteria were as follows: corpses with collections of fluids within the lateral brain ventricles, the thoracic cavities, and abdominal cavities as well as the pericardial sac and the left and right heart ventricles. Blood, serous fluids, and CSF were assessed only in fresh corpses with a postmortem interval (PMI) of 1 to 4 days (time between death and MRI scan). Putrefied CSF was assessed in putrefied corpses. Putrefaction was determined when there were relevant external signs (brown- to greencolored skin veins, ablation of external skin layers, and vesicle formations of the skin) and internal signs of putrefaction. The internal state of decomposition was determined with the radiological alteration index (RAI) from Egger et al. using whole-body computed tomography prior to PMMRI. Only putrefied corpses with a RAI over 60 were included in this study $[25,26]$. The PMI of the putrefied corpses ranged from 3 weeks to 3.5 months.

\section{MRI, temperature monitoring, and image analysis}

For MRI scanning (Siemens Magnetom Symphony Tim $1.5 \mathrm{~T}$ ), the corpses were wrapped in an artifact-free body bag or a linen sheet. All subjects were scanned in supine position. The MRI quantification sequence was a multi-slice turbo spin echo (TSE) sequence, where each acquisition was performed with two different echo-times, at 20 and $95 \mathrm{~ms}$. Four different saturation delays times were acquired, at 100, 400,1100 , and $2400 \mathrm{~ms}$, using a TR of $2500 \mathrm{~ms}$. The scan time was $15 \mathrm{~min}$ for each brain or heart. In this setup, eight different images were acquired, with different effects of $T_{1}$ and $T_{2}$ relaxation. The $T_{1}$ and $T_{2}$ relaxation, as well as proton density (PD), was retrieved by a commercially available post-processing tool (SyMRI Autopsy, SyntheticMR, Linköping, Sweden) [27]. The same software provided the synthetic $T_{1}$-weighted, $T_{2}$-weighted, and PD images for visual support. Twenty to 24 cardiac short axis slices or brain slices of $4 \mathrm{~mm}$ in thickness were acquired with a gap of $0.3 \mathrm{~mm}$. The use of the image data for the present study was approved by the local ethics committee.

During the MRI scan, the corpse temperature was assessed in real time with MR-compatible temperature probes that were placed in the esophagus. The temperatures of the fresh corpses ranged from $4-33{ }^{\circ} \mathrm{C}$ (mean temperature $19.3{ }^{\circ} \mathrm{C}$, SD 6.9), and the temperature of the putrefied corpses ranged from 16$21^{\circ} \mathrm{C}$ (mean temperature $22.7^{\circ} \mathrm{C}, \mathrm{SD} 4.8$ ).

To obtain quantitative values of fluids, a total of five independent and non-overlapping square regions of interest (ROIs) were placed in three different slices of cardiac short axis MRI images or axial brain MRI images (Fig. 1) by one observer experienced in forensic imaging. The ROIs were placed over the entire ventro-dorsal expansion of the fluid collections. The sizes of the ROIs depended on the extensions of the fluid collections but were at least $0.5 \mathrm{~cm}$ in each square dimension. In cases of visible segmentation, the upper and lower layers were measured separately.

The quantitative $T_{1}, T_{2}$, and PD values of serous fluids, blood, and CSF were 3D plotted and related to the body core temperature of the corpse at the time of data acquisition. Because of the low temperature range $\left(16-21^{\circ} \mathrm{C}\right)$ of the putrefied corpses, the putrefied CSF was not related to the body core temperature. 
Fig. 1 Synthetically quantified cardiac short axis $T_{2}$ w MRI image. Quantitative values (red frame upper right corner) were obtained by placing ROIs (yellow arrow, example measurement shown for a serous fluid collection in the pericardial sac) on detected fluid collections

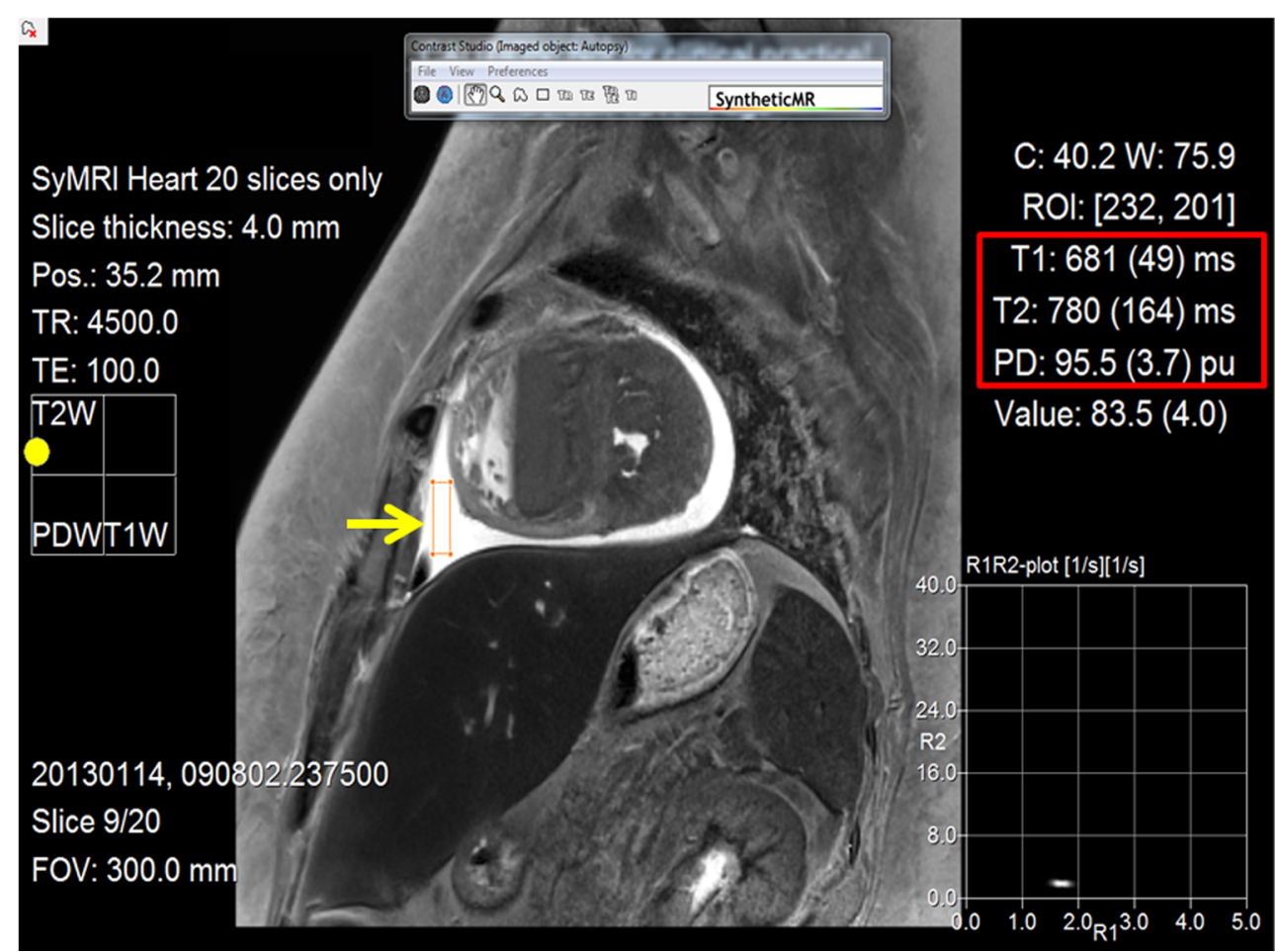

The ThreeDify Excel Grapher Add-in for Microsoft Excel $^{\circledR}$ was applied to visualize clustering and separation of blood, serous fluids, CSF, and putrefied CSF in 3D plots.

\section{Macroscopic analysis of sampled fluids}

Autopsies were authorized by the local authorities and performed by forensic pathologists immediately after MRI scanning or on the following day. The fluid mediums that were measured in the MRI images were obtained at autopsy, and their macroscopic appearance (viscosity, color, and clarity) was examined immediately afterwards. Low viscosity fluids that were light yellow to light red in color and clear or slightly unclear in appearance were characterized as serous fluids in the thoraco-abdominal compartments or CSF in the lateral brain ventricles, respectively. Blood was characterized by its typical red appearance and viscosity. Blood clots were characterized as being compact or thickened blood formations of a red to yellowish color within the right and left heart ventricles. Only blood clots from the heart ventricles were assessed for this study. The lower cellular layers of sedimented blood formations were not considered blood clots. Fluids in the ventricles of putrefied corpses that were dark red to black in color and unclear in appearance were characterized as putrefied CSF. In 7 cases of the 15 putrefied corpses, the macroscopic evaluation of the fluids in the lateral ventricles was not possible because of the highly softened or liquefied consistency of the brain tissues.

At autopsy, fluid mediums were obtained from the following body cavities: serous fluids from the thoracic cavities $(n=12)$ and the pericardial sac $(n=13)$; fluid blood from the right heart and left heart ventricles $(n=12)$, sedimented blood from the pericardial sac $(n=2)$, thoracic cavities $(n=6)$, abdominal cavity $(n=2)$, and left and right heart ventricles $(n=$ 18 ); blood clots from the right and left heart ventricles $(n=13)$; CSF from the lateral brain ventricles $(n=16)$; and putrefied CSF from the lateral brain ventricles $(n=15)$.

\section{Generation of equations for temperature correction and statistical analysis}

Using Microsoft Excel ${ }^{\circledR}$, linear equations were generated to assess the $T_{1} /$ temperature, $T_{2} /$ temperature, and $\mathrm{PD} /$ temperature relationships for each investigated fluid medium and blood clots. Those equations were used to correct the values of $T_{1}, T_{2}$, and PD: the according corpse temperatures were subtracted from $37{ }^{\circ} \mathrm{C}$ ( $\Delta$ temperatures). $\Delta$ temperatures were applied in the equations generated from the quantitative values/temperature relation graphs to gain $\Delta T_{1}, \Delta T_{2}$, and $\Delta \mathrm{PD}$. Those $\Delta$ values were summated to the uncorrected $T_{1}, T_{2}$, and PD values to gain the temperature corrected values. Temperature corrections were conducted for serous fluids, blood, blood clots and CSF to a temperature of $37^{\circ} \mathrm{C}$. Due to the small body temperature ranges of the putrefied corpses, no equations for putrefied CSF were generated. Thus, no temperature corrections for the quantitative $T_{1}, T_{2}$, and PD values of putrefied CSF were conducted.

SPSS ${ }^{\circledR}$ was used to analyze quantitative sample data of $T_{1}$, $T_{2}$, and PD values. The samples were tested for normality using the Kolmogorov-Smirnov test and the Shapiro-Wilk 
test. Both tests showed multiple samples that were not normally distributed. Paired Kruskal-Wallis tests were performed to determine significant differences between the samples. Bonferroni's correction was performed to evaluate significant differentiability of quantitative $T_{1}, T_{2}$, and PD values between serous fluids, fluid blood, sedimented blood, blood clots, CSF, and putrefied CSF.

Using the MATLAB ${ }^{\circledR}$ software, the receiver operator characteristic (ROC) curve approach was applied to give accuracy of discrimination between the tested fluids. Accuracy was measured by the area under the ROC curve (AUC) with the traditional academic point system: $0.90-1=$ excellent; .80 $0.90=$ good; $.70-0.80=$ fair; $0.60-0.70=$ poor; $0.50-0.60=$ fail.

\section{Results}

\section{Quantitative values/temperature relations}

The quantitative values/temperature relations obtained in the investigated cases were expressed by linear equations. Table 1 lists the linear equations generated for serous fluids, fluid blood, sedimented blood, blood clots, and CSF. Individual variations in the relationship between the change in temperature and the change in quantitative values were observed in all investigated mediums. A temperature dependence was observed mainly for the $T_{1}$ and the $T_{2}$ values of fluid mediums as shown in the example of the plasma layer of sedimented blood in Fig. 2. In the mediums that mainly consisted of red blood cells (blood clots and the erythrocyte layer of sedimented blood), $T_{2}$ values did not exhibit a relevant temperature dependence. The PD values were only minimally influenced by temperature in all investigated mediums. Temperature correction to a temperature of $37^{\circ} \mathrm{C}$ resulted in lower standard deviations in all investigated mediums.

\section{Differentiation of blood, serous fluids, CSF, and putrefied CSF with quantitative values}

Table 2 shows the mean quantitative values $\left(T_{1}\right.$ and $T_{2}$ relaxation times as well as PD) obtained for serous fluids, fluid blood, sedimented blood, blood clots, CSF, and putrefied CSF in 86 forensic cases. All fluid mediums except the putrefied CSF $(n=15)$ were correlated with body temperature at the time of MRI scanning. Statistical analysis revealed that temperature correction had no significant influence on the differentiability of all investigated fluids. Quantitative values were plotted in a 3D-coordinate system and are depicted with and without correction for a temperature of $37^{\circ} \mathrm{C}$ (Fig. 3). In the $3 \mathrm{D}$ plot as well as in statistical analysis, the quantitative values of serous fluids and CSF could be well discriminated from the values of fluid blood, sedimented blood and blood clots. Putrefied CSF could be differentiated from all other investigated fluid mediums. The quantitative values of the serous fluids in the pericardial sac clearly differed from those of the serous fluids in the thoracic cavities in the 3D plot. The quantitative values of fluid blood, sedimented blood, and blood clots were all distinguishable from each other in the 3D plot as well as in statistical analysis. Furthermore, the investigated blood fractions differed significantly from blood clots (Fig. 3 and Table 3).

\section{Discussion}

In the present postmortem study, the synthetic MRI approach was applied for the first time to investigate different fluids within the body. Serous fluids, fluid blood, sedimented blood, blood clots, CSF, and putrefied CSF could be well differentiated from each other by their quantitative values of $T_{1}, T_{2}$, and $\mathrm{PD}$. This provides the basis for further promising applications of quantitative MRI. Databases for quantitative values of $T_{1}$, $T_{2}$, and PD of fluids can be established. As a result, a scale of quantitative values for fluids can be created similar to the Hounsfield scale in CT applications. Using quantitative data from synthetic MRI applications also provides the basis for automatic fluid detection and computer-aided diagnosis. Synthetic MRI images can be loaded on a personal computer with properly adapted software. Then, computer-aided diagnostics can be implemented based on established values of the signal behavior of the fluid mediums [28-30]. For example, blood could automatically be detected and encoded in color. This could help the image reader detect hemorrhages and

Table 1 Linear equations for fluids and blood clots generated from the relation of $T_{1}, T_{2}$, and PD to body core temperature at the time of scanning as obtained from 71 forensic cases

\begin{tabular}{lllllll}
\hline & Fluid blood & Blood plasma layer & Erythrocyte layer & Blood clot & Serous fluids & CSF \\
\hline$T_{1}$ /temperature & $T_{1}=6.63 x+507$ & $T_{1}=4.93 x+562$ & $T_{1}=7.8 x+449$ & $T_{1}=4.63 x+454$ & $T_{1}=3.9 x+819$ & $T_{1}=4.65 x+585$ \\
$T_{2} /$ temperature & $T_{2}=7.42 x+159$ & $T_{2}=8.93 x+239$ & $T_{2}=-1.05 x+79$ & $T_{2}=1.69 x+70$ & $T_{2}=23.01 x+479$ & $T_{2}=32.27 x+418$ \\
PD/temperature & $\mathrm{PD}=0.43 x+74$ & $\mathrm{PD}=0.44 x+82$ & $\mathrm{PD}=0.43 x+68$ & $\mathrm{PD}=0.44 x+66$ & $\mathrm{PD}=-0.59 x+101$ & $\mathrm{PD}=0.19 x+82$ \\
\hline
\end{tabular}

Due to small temperature ranges, no equations for putrefied CSF were generated. ( $T_{1}: T_{1}$ relaxation time in milliseconds; $T_{2}: T_{2}$ relaxation time in milliseconds; $T$ : body core temperature in ${ }^{\circ} \mathrm{C}$; PD: PD in $\%$ related to pure water $(100 \%)$ ) 
Blood plasma layer T1 $y=4.93 x+562$

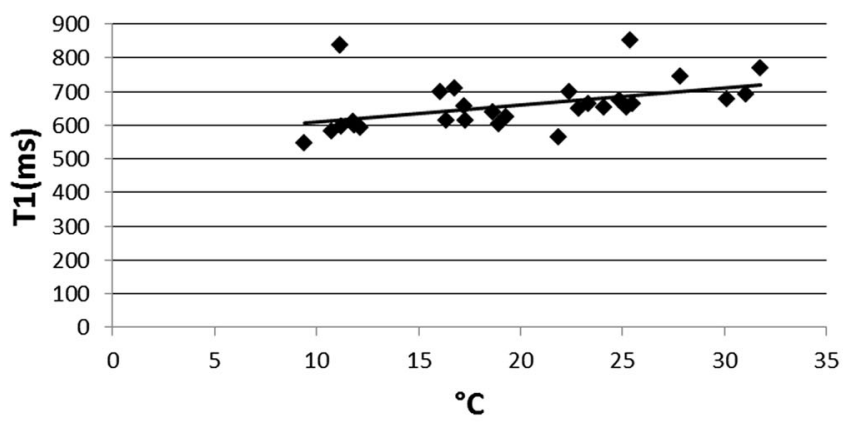

Blood plasma layer T2 $y=8.93 x+239$

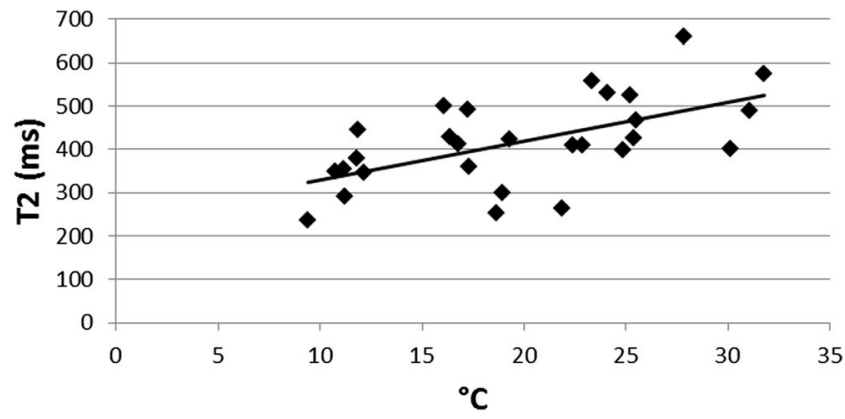

Blood plasma layer PD $y=0.44 x+82$

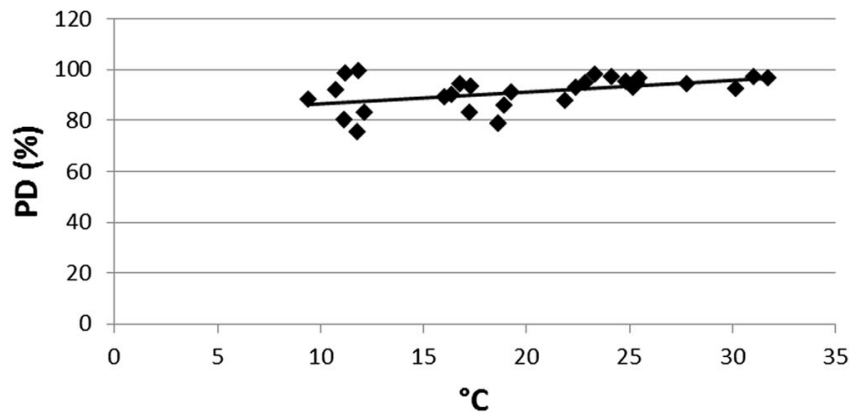

Fig. 2 Sample plots of the $T_{1}$ and $T_{2}$ relaxation times and PD of the plasma layer of sedimented blood vs. body core temperature at the time of scanning. Note that there is a temperature dependence mainly for the $T_{1}$ and $T_{2}$ values. It was assumed that the quantitative $T_{1}, T_{2}$, and PD values

discriminate blood from other fluid mediums in the corpse. The detection of blood collections may also be relevant in the decision to perform postmortem CT-angiography to detect the source of exsanguination [31, 32].

The temperature dependence of the quantitative values challenges the postmortem synthetic MRI approach. In the present study, this temperature dependence was observed mainly for the $T_{1}$ and $T_{2}$ relaxation times in serous fluids, blood, and blood clots. Preliminary studies demonstrated a temperature dependence of $T_{1}$ relaxation times in blood. It was shown that the $T_{1}$ relaxation time correlated with rising correlate linearly with rising temperature. Linear equations for temperature correction are depicted on the upper right corner of each table

temperature [33-39]. Our data confirmed a correlation between the $T_{1}$ values and rising temperature. A new finding we observed was a relevant $T_{2}$ temperature dependence for fluids as well. In forensic practice, corpses are scanned with temperatures usually ranging between 0 and $40{ }^{\circ} \mathrm{C}$. To compare quantitative MRI data from different corpse scans, the temperature dependence of the quantitative values has to be taken into account. A valid comparison of different MRI scans is possible only when the quantitative values gained from the scans are corrected for temperature. Then, the temperaturecaused differences between individual corpses can be

Table 2 Mean quantification values ( $T_{1}$ and $T_{2}$ in ms; PD in \%) and $95 \%$ confidence intervals $(\mathrm{CI})$ of the investigated fluids and blood clots obtained from 5 measurements in 86 forensic cases

\begin{tabular}{|c|c|c|c|c|c|c|}
\hline & $T_{1}$ uncorrected $(\mathrm{CI})$ & $\begin{array}{l}T_{1} \text { corrected } \\
\text { to } 37^{\circ} \mathrm{C}(\mathrm{CI})\end{array}$ & $T_{2}$ uncorrrected $(\mathrm{CI})$ & $\begin{array}{l}T_{2} \text { corrected } \\
\text { to } 37^{\circ} \mathrm{C}(\mathrm{CI})\end{array}$ & PD uncorrected (CI) & $\begin{array}{l}\text { PD corrected } \\
\text { to } 37^{\circ} \mathrm{C}(\mathrm{CI})\end{array}$ \\
\hline Fluid blood & $659(18)$ & 805 (19) & $492(12)$ & $472(11)$ & $82(3)$ & $90(4)$ \\
\hline Blood plasma layer & $660(27)$ & $744(24)$ & 417 (29) & $570(31)$ & $91(6 ; 2)$ & $99(2)$ \\
\hline Erythrocyte layer & $608(27)$ & $742(19)$ & $57(6)$ & $39(5)$ & $76(2)$ & $84(2)$ \\
\hline Blood clot & $538(26)$ & $625(19)$ & $101(12)$ & $133(11)$ & $74(3)$ & $82(3)$ \\
\hline Serous fluids & $752(41)$ & $746(47)$ & $850(124)$ & $1293(107)$ & $92(3)$ & $81(6)$ \\
\hline $\mathrm{CSF}$ & $695(43)$ & 757 (22) & $1052(151)$ & $1612(27)$ & $86(5)$ & $89(5)$ \\
\hline Putrefied CSF & $578(22)$ & - & $231(17)$ & - & $92(4)$ & - \\
\hline
\end{tabular}

Data are presented with (except for putrefied CSF) and without correction to $37^{\circ} \mathrm{C}$ 
equalized. In the present study, linear equations to correct for the temperature dependence of $T_{1}, T_{2}$, and PD quantitative values of fluid mediums to $37{ }^{\circ} \mathrm{C}$ were introduced. The correction of quantitative values to $37^{\circ} \mathrm{C}$ resulted in slightly better discrimination between the fluid mediums in the $3 \mathrm{D}$ plot as well as in the statistical analyses, which supports the assertion that temperature correction is needed.

The present study has several limitations. Measurements were taken by only one observer, and no statistical reproducibility analysis of quantitative value measurement was conducted. It is likely to expect that there would be a variability of quantitative values between different observers. However, the conducted measurement technique is relatively easy to perform and the results of the present study rather do not indicate that the expected inter observer variability would have relevant influence on the differentiability of fluids. Furthermore for some fluids, only small numbers could be assessed. To create databases with regular quantitative $T_{1}$, $T_{2}$, and PD values for different fluids and blood, a larger number of cases will be required. Additionally, the investigated fluids and blood were not examined histologically or chemically. We observed differences in the quantitative values of serous fluids in the pericardial sac compared with serous fluids in the thoracic cavities and CSF in the lateral brain ventricles. It can be assumed that different protein densities and cellular impurities are responsible for the differing quantitative values in serous fluids and CSF [26, 40-42]. The possibility of detecting changes in cellular components and protein density in serous fluids and CSF based on quantitative values may be of relevant interest because a measurable change in the chemical composition of fluids may be a noninvasive indicator of pathologic conditions such as inflammation or tumors. It is noteworthy that quantitative MRI is not the only promising approach to discern various fluid types. Clinical studies showed that it is possible to differentiate serous and purulent infectious fluids by means of diffusion-weighted MRI [43, 44]. Other approaches such as the application of magnetization transfer ratio and NMR spectroscopy are also potentially feasible to discriminate different fluids types [45-48].

Interestingly, we were able to observe that differentiation between the cellular layers of sedimented blood and blood clots was possible based on their quantitative values. This may be explained by different contents of proteins in the coagulation cascade, activation of platelets and platelet factors, and arrangement of cellular components within the cellular layer of sedimented blood compared with blood clots. If quantitative MRI is feasible for detecting different protein contents in mediums consisting mainly of blood cells, it might also be used to differentiate between vital thrombi and postmortem blood clots. So far, the gold standard for recognizing a vital thrombus is histologic diagnosis based on the layered arrangement of cellular components and the existence of cross-linked fibrin meshes $[42,49]$. Jackowski et al. demonstrated that
Fig. 3 Three defined views (PD/ $T_{1}$ view, $T_{2} / T_{1}$ view, and $\mathrm{PD} / T_{2}$ view) on a $3 \mathrm{D}$ plot of fluids and blood clots based on $T_{1}$ and $T_{2}$ relaxation times and PD. Values are depicted as not corrected for temperature (a) and corrected for a temperature of $37^{\circ} \mathrm{C}(\mathbf{b})$. The serous fluids from the thoracic cavities and pericardial sac are depicted with different colors. Note that the clusters of CSF and serous fluids on the one hand and fluid blood and sedimented blood on the other hand can be well differentiated from each other. The depicted values corrected for a temperature of $37^{\circ} \mathrm{C}$ allow for better discrimination between the single fluids, which can be seen best in the $T_{1} / T_{2}$ and $\mathrm{PD} / T_{2}$ views. Putrefied CSF can be differentiated from all other investigated fluids in the plots that were not corrected for temperature (due to small temperature ranges, putrefied CSF was not corrected for temperature)

conventional unenhanced 3-T MRI is also feasible for detecting pulmonary thromboembolism via the changes in signal intensity of thrombi in conventional MRI images [50]. However, diagnosis of pulmonary thromboembolism in conventional MRI images is always challenging and often inconclusive. Further investigations that compare quantitative values of vital thrombi and postmortem blood clots are necessary.

For the first time, quantitative values of putrefied fluids were evaluated. Based on their quantitative values, the investigated putrefied fluids in the lateral brain ventricles could be clearly differentiated from the CSF in the lateral brain ventricles as well as from all other investigated fluids of fresh corpses. However, the quantitative values of putrefied thoraco-abdominal fluids were not assessed. It may be possible that these values differ from the putrefied CSF values due to differing tissue compositions of thoraco-abdominal organs and fluids [42]. If the quantitative values of putrefied fluids are known entirely, they may be used to discriminate the fluids from relevant forensic ante mortem pathologies. The results of the present study indicate that, for example, antemortem hemorrhages in putrefied corpses could be detected and characterized based on their quantitative values. Further investigations and assessment of putrefied brain tissues as well as of putrefied thoraco-abdominal organs, fluids, and pathologies are necessary.

A 1.5-T MR scanner was used for the present study. Because the relaxation times depend on the strength of the magnetic field, it is reasonable to expect that the use of MR scanners with different magnetic field strengths, such as $3 \mathrm{~T}$, will result in different quantitative values for $T_{1}$ and $T_{2}$ for the same fluids and temperatures [16]. Therefore, it is inevitable that the quantitative values of the relaxation times of fluids have to be adapted not only to different temperatures but also to different magnetic field strengths.

\section{Conclusions}

Postmortem 1.5-T MR quantification using a combination of the relaxation times of $T_{1}$ and $T_{2}$ and the PD value allows for reliable discrimination and characterization of serous fluids, 
Not corrected for temperature

PD

a

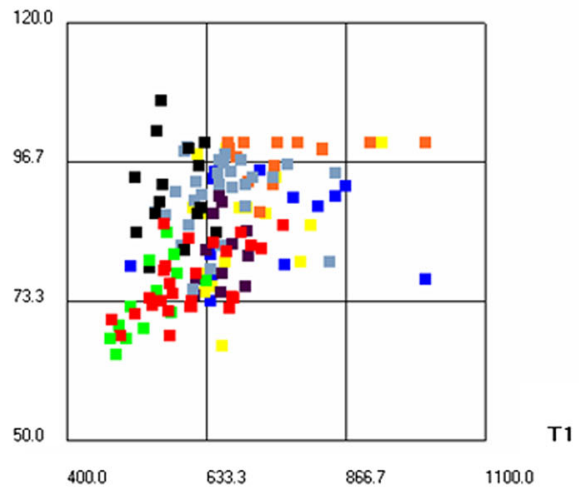

Corrected to $37^{\circ} \mathrm{C}$

PD

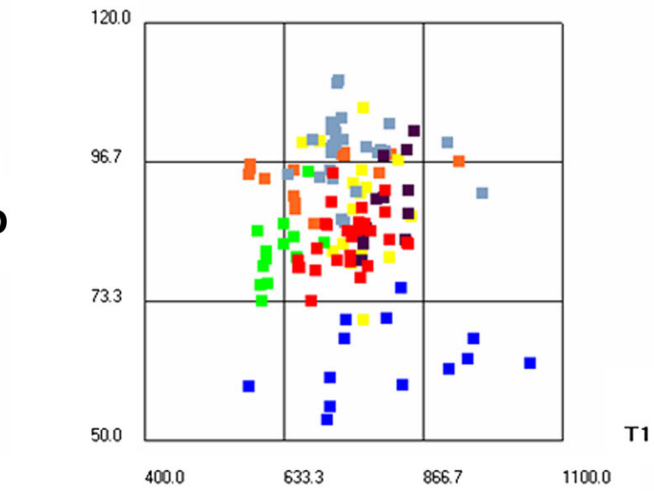

Blood clot OBlood plasma layer OCSF OErythrocyte layer - Fluid blood P Putrefied CSF Serous fluids pericardial OSerous fluids thoracic

Not corrected for temperature

T2

a

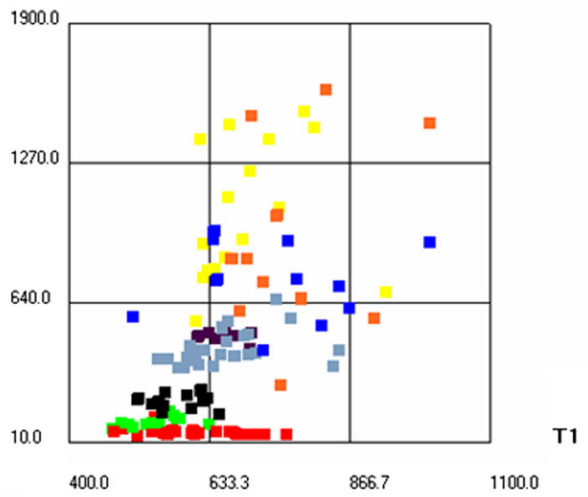

Corrected to $37^{\circ} \mathrm{C}$

T2

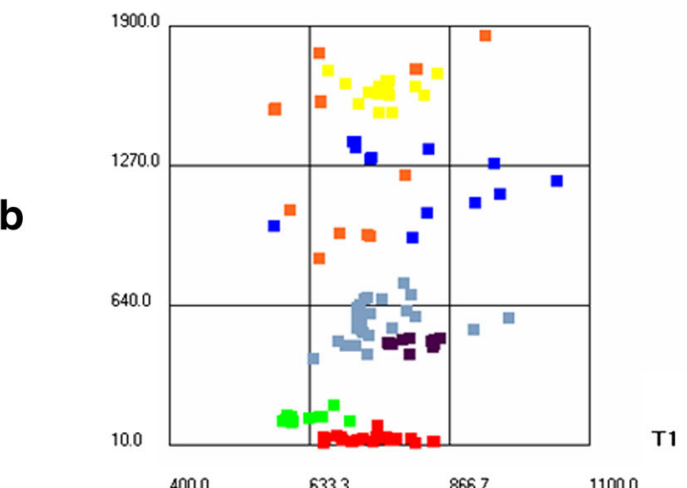

Blood clot OBlood plasma layer OCSF Erythrocyte layer 9 Fluid blood Putrefied CSF Serous fluids pericardial O Serous fluids thoracic

Not corrected for temperature

PD

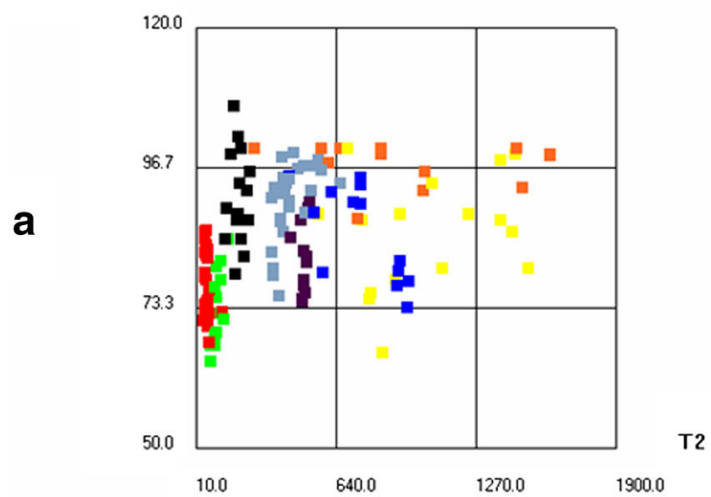

Corrected to $37^{\circ} \mathrm{C}$

PD

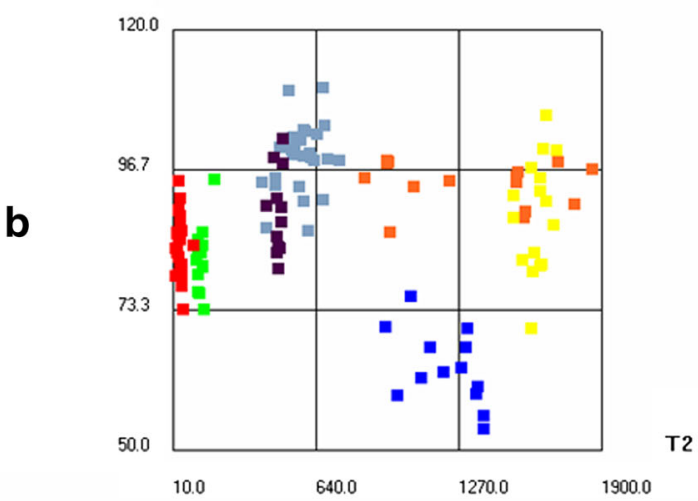

O Blood clot OBlood plasma layer OCSF O Erythrocyte layer O Fluid blood Putrefied CSF Serous fluids pericardial O Serous fluids thoracic 


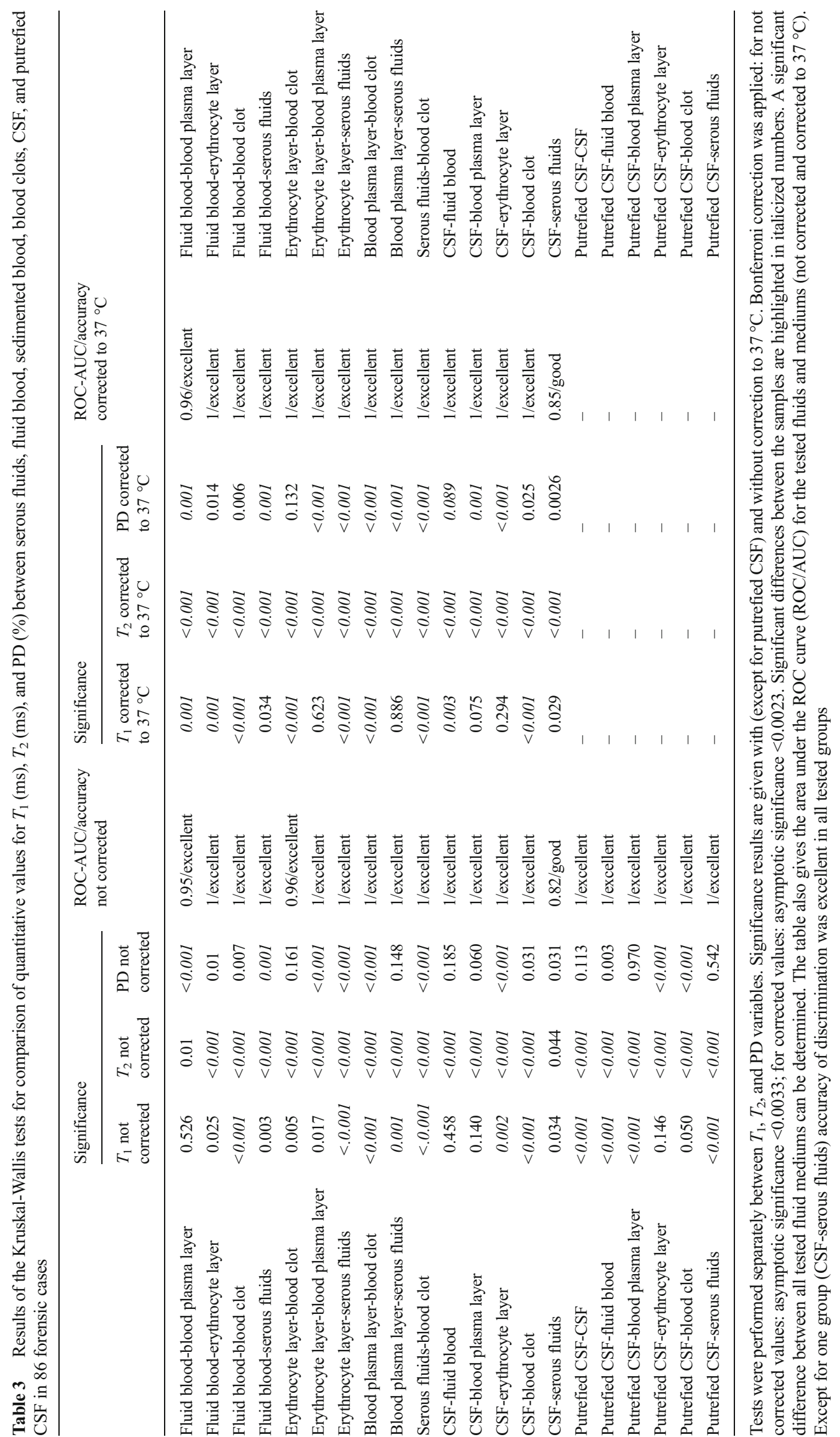


fluid blood, sedimented blood, blood clots, CSF, and putrefied CSF. The quantification approach allows for computer-aided detection and diagnosis of hemorrhages. The quantitative values of mainly $T_{1}$ and $T_{2}$ were temperature dependent. Equations to correct for the temperature dependence at $1.5 \mathrm{~T}$ have been introduced.

Acknowledgments The authors would like to thank the team of forensic pathologists and forensic autopsy technicians at the Institute of Forensic Medicine Bern for their support in handling the cases.

\section{References}

1. Lundström C, Persson A, Ross S et al (2012) State-of-the-art of visualization in post-mortem imaging. APMIS 120:316-326

2. Jackowski C, Schwendener N, Grabherr S, Persson A (2013) Postmortem cardiac 3T magnetic resonance imaging: visualizing the sudden cardiac death? J Am Coll Cardiol 62(7):617-629

3. Roberts IS, Benbow EW, Bisset R et al (2003) Accuracy of magnetic resonance imaging in determining cause of sudden death in adults: comparison with conventional autopsy. Histopathology 42 : 424-430

4. Thali MJ, Yen K, Schweitzer W et al (2003) Virtopsy, a new imaging horizon in forensic pathology: virtual autopsy by postmortem multislice computed tomography (MSCT) and magnetic resonance imaging (MRI) — a feasibility study. J Forensic Sci 48(2):386-403

5. Dirnhofer R, Jackowski C, Vock P, Potter K, Thali MJ (2006) VIRTOPSY: minimally invasive, imaging-guided virtual autopsy. Radiographics 26(5):1305-1333

6. Patriquin L, Kassarjian A, Barish M et al (2001) Postmortem whole-body magnetic resonance imaging as an adjunct to autopsy: preliminary clinical experience. J Magn Reson Imaging 13:277287

7. Brogdon BG (2010) Brogdon's forensic radiology, 2nd edn. CRC Press, Boca Raton

8. Aghayev E, Christe A, Sonnenschein M, Yen K, Jackowski C, Thali MJ, Dirnhofer R, Vock P (2008) Postmortem imaging of blunt chest trauma using CT and MRI: comparison with autopsy. J Thorac Imaging 23(1):20-27

9. Bolliger SA, Thali MJ, Aghayev E, Jackowski C, Vock P, Dirnhofer R, Christe A (2007) Postmortem noninvasive virtual autopsy: extrapleural hemorrhage after blunt thoracic trauma. Am J Forensic Med Pathol 28(1):44-47

10. Aghayev E, Sonnenschein M, Jackowski C et al (2006) Postmortem radiology of fatal hemorrhage: measurements of cross-sectional areas of major blood vessels and volumes of aorta and spleen on MDCT and volumes of heart chambers on MRI. AJR 187:209-215

11. Montano-Loza AJ (2013) New concepts in liver cirrhosis: clinical significance of sarcopenia in cirrhotic patients. Minerva Gastroenterol Dietol 59(2):173-186

12. Treglia G, Sadeghi R, Annunziata S, Lococo F, Cafarotti S, Bertagna F, Prior JO, Ceriani L, Giovanella L (2014) Diagnostic accuracy of (18)F-FDG-PET and PET/CT in the differential diagnosis between malignant and benign pleural lesions: a systematic review and meta-analysis. Acad Radiol 21(1):11-20

13. Solooki M, Miri M (2013) Approach to undiagnosed exudative pleural effusion: the diagnostic yield of blind pleural biopsy. Caspian J Intern Med 4(2):642-647

14. Huang XE, Wei GL, Huo JG, Wang XN, Lu YY, Wu XY, Liu J, Xiang J, Feng JF (2013) Intrapleural or intraperitoneal lobaplatin for treatment of patients with malignant pleural effusion or ascites. Asian Pac J Cancer Prev 14(4):2611-2614
15. Adhikari P, Pathak UN, Uprety D, Sapkota S (2012) Profile of ascites patient admitted in Nepal Medical College Teaching Hospital. Nepal Med Coll J 14(2):111-113

16. Haacke ME, Brown RW, Thompson MR, Venkatesh N (1999) Magnetic resonance imagingphysical principles and sequence design. Wiley, New York

17. Jackowski C, Thali M, Aghayev E, Yen K, Sonnenschein M, Zwygart K, Dirnhofer R, Vock P (2006) Postmortem imaging of blood and its characteristics using MSCT and MRI. Int J Legal Med 120(4):233-240

18. Schleyer F (1958) Postmortem blood viscosity, blood cell volume, osmotic erythrocyte resistance and blood sedimentation in relation to cadaver age and cause of death. Virchows Arch 331(3):276-286

19. Shiono T, Yoshikawa K, Takenaka E, Hisamatsu K (1993) MR imaging of pleural and peritoneal effusion. Radiat Med 11(4): $123-126$

20. Aprile I, Iaiza F, Lavaroni A, Budai R, Dolso P, Scott CA, Beltrami CA, Fabris G (1999) Analysis of cystic intracranial lesions performed with fluid-attenuated inversion recovery MR imaging. AJNR Am J Neuroradiol 20(7):1259-1267

21. Warntjes JB, Dahlqvist O, Lundberg P (2007) Novel method for rapid, simultaneous $\mathrm{T} 1, \mathrm{~T}^{*} 2$, and proton density quantification. Magn Reson Med 57:528-537

22. Warntjes JB, Leinhard OD, West J, Lundberg P (2008) Rapid magnetic resonance quantification on the brain: optimization for clinical usage. Magn Reson Med 60:320-329

23. Warntjes MJ, Kihlberg J, Engvall J (2010) Rapid T1 quantification based on 3D phase sensitive inversion recovery. BMC Med Imaging 10:19

24. Blystad I, Warntjes JB, Smedby O, Landtblom AM, Lundberg P, Larsson EM (2012) Synthetic MRI of the brain in a clinical setting. Acta Radiol 53:1158-1163

25. Egger C, Vaucher P, Doenz F, Palmiere C, Mangin P, Grabherr S (2012) Development and validation of a postmortem radiological alteration index: the RA-Index. Int J Legal Med 126(4):559-566

26. Zech WD, Jackowski C, Buetikofer Y, Kara L (2014) Characterization and differentiation of body fluids, putrefaction fluid, and blood using Hounsfield unit in postmortem CT. Int J Legal Med 128(5):795-802

27. Synthetic MR products website. Available at: http://www. syntheticmr.com. Accessed 30 June 2014

28. Ljung P, Winskog C, Persson A, Lundström C, Ynnerman A (2006) Full body virtual autopsies using a state-of-the-art volume rendering pipeline. IEEE Trans Vis Comput Graph 12:869-876

29. Jackowski C, Warntjes MJ, Kihlberg J, Berge J, Thali MJ, Persson A (2011) Quantitative MRI in isotropic spatial resolution for forensic soft tissue documentation. Why and how ? J Forensic Sci 56: 208-215

30. Persson A, Lindblom M, Jackowski C (2011) A state-of-the-art pipeline for postmortem $\mathrm{CT}$ and MRI visualization: from data acquisition to interactive image interpretation at autopsy. Acta Radiol 52:522-536

31. Grabherr S, Grimm J, Dominguez A, Vanhaebost J, Mangin P (2014) Advances in postmortem CT-angiography. Br J Radiol 87(1036):20130488

32. Jackowski C, Persson A, Thali MJ (2008) Whole body postmortem angiography with a high viscosity contrast agent solution using poly ethylene glycol as contrast agent dissolver. J Forensic Sci 53(2):465-468

33. Dickinson RJ, Hall AS, Hind AJ, Young IR (1986) Measurement of changes in tissue temperature using MR imaging. J Comput Assist Tomogr 10:468-472

34. Wlodarczyk W, Hentschel M, Wust P et al (1999) Comparison of four magnetic resonance methods for mapping small temperature changes. Phys Med Biol 44:607-624 
35. Peller M, Kurze V, Loeffler R et al (2003) Hyperthermia induces T1 relaxation and blood flow changes in tumors. A MRI thermometry study in vivo. Magn Reson Imaging 21:545-551

36. Parker DL, Smith V, Sheldon P, Crooks LE, Fussell L (1983) Temperature distribution measurements in two-dimensional NMR imaging. Med Phys 10:321-325

37. Bertsch F, Mattner J, Stehling MK et al (1998) Non-invasive temperature mapping using MRI: comparison of two methods based on chemical shift and T1-relaxation. Magn Reson Imaging 16:393-404

38. Youl BD, Hawkins CP, Morris JK, DuBoulay EP, Tofts PS (1992) In vivo T1 values from guinea pig brain depend on body temperature. Magn Reson Med 24:170-173

39. Ruder TD, Hatch GM, Siegenthaler L et al (2012) The influence of body temperature on image contrast in post mortem MRI. Eur J Radiol 81:1366-1370

40. Block DR, Algeciras-Schimnich A (2013) Body fluid analysis: clinical utility and applicability of published studies to guide interpretation of today's laboratory testing in serous fluids. Crit Rev Clin Lab Sci 50(4-5):107-124

41. Bonnema J, Ligtenstein DA, Wiggers T, van Geel AN (1999) The composition of serous fluid after axillary dissection. Eur J Surg 165(1):9-13

42. Brinkmann B, Madea B (2004) Handbuch gerichtliche Medizin, vol 1, 1st edn. Springer, Berlin

43. Spüntrup E, Bücker A, Adam G, van Vaals JJ, Günther RW (2001) Differentiation of serous and purulent fluids in vitro and in vivo by means of diffusion-weighted MRI. Röfo 173(1):65-71
44. Gaviani P, Schwartz RB, Hedley-Whyte ET, Ligon KL, Robicsek A, Schaefer P, Henson JW (2005) Diffusion-weighted imaging of fungal cerebral infection. AJNR Am J Neuroradiol 26(5):11151121

45. Jia G, Takayama Y, Flanigan DC, Kaeding CC, Zhou J, Chaudhari A, Clark D et al (2011) Quantitative assessment of mobile protein levels in human knee synovial fluid: feasibility of chemical exchange saturation transfer (proteinCEST) MRI of osteoarthritis. Magn Reson Imaging 29(3):335-341

46. Mishra AM, Reddy SJ, Husain M, Behari S, Husain N, Prasad KN, Kumar S, Gupta RK (2006) Comparison of the magnetization transfer ratio and fluid-attenuated inversion recovery imaging signal intensity in differentiation of various cystic intracranial mass lesions and its correlation with biological parameters. J Magn Reson Imaging 24(1):52-56

47. Kumar V, Dwivedi DK, Jagannathan NR (2014) High-resolution NMR spectroscopy of human body fluids and tissues in relation to prostate cancer. NMR Biomed 27(1):80-89

48. Lam CW, Law CY (2014) Pleural effusion lipoproteins measured by NMR spectroscopy for diagnosis of exudative pleural effusions: a novel tool for pore-size estimation. J Proteome Res 13(9):4104 4112

49. Bohm E, Hochkirchen KH (1983) Ultrastructure of intravital, postmortem and autolysed fibrin. Forensic Sci Int 21:117-127

50. Jackowski C, Grabherr S, Schwendener N (2013) Pulmonary thrombembolism as cause of death on unenhanced postmortem 3T MRI. Eur Radiol 23(5):1266-1270 